\title{
Morphology, mechanical characterization and in vivo neo-vascularization of chitosan particle aggregated scaffolds architectures
}

\author{
Patrícia B. Malafaya ${ }^{\text {a,b,* }}$,írcia C. Santos ${ }^{\mathrm{a}, \mathrm{b}}$, Martijn van Griensven ${ }^{\mathrm{c}}$, Rui L. Reis ${ }^{\mathrm{a}, \mathrm{b}}$ \\ a 3B's Research Group - Biomaterials, Biomimetics and Biodegradables, Department of Polymer Engineering, University of Minho, Campus de Gualtar, 4710-057 Braga, Portugal \\ ${ }^{\mathrm{b}}$ IBB - Institute for Biotechnology and Bioengineering, PT Government Associated Laboratory, Braga, Portugal \\ ${ }^{\mathrm{c}}$ Ludwig Boltzmann Institute for Experimental and Clinical Traumatology, Austrian Cluster for Tissue Regeneration, Donaueschingenstrasse 13, A-1200 Vienna, Austria
}

\section{A R T I C L E I N F O}

\section{Article history:}

Received 9 April 2008

Accepted 13 June 2008

Available online 22 July 2008

\section{Keywords:}

Scaffolds

Particle aggregation

Chitosan

Micro-Computed Tomography (micro-CT)

Dynamical Mechanical Analysis (DMA)

In vivo response

\begin{abstract}
A B S T R A C T
The present study intended to evaluate the performance of chitosan-based scaffolds produced by a particle aggregation method aimed to be used in tissue engineering applications addressing key issues such as morphological characteristics, mechanical performance and in vivo behaviour. It is claimed that the particle aggregation methodology may present several advantages, such as combine simultaneously a high interconnectivity with high mechanical properties that are both critical for an in vivo successful application. In order to evaluate these properties, micro-Computed Tomography (micro-CT) and Dynamical Mechanical Analysis (DMA) were applied. The herein proposed scaffolds present an interesting morphology as assessed by micro-CT that generally seems to be adequate for the proposed applications. At a mechanical level, DMA has shown that chitosan scaffolds have an elastic behaviour under dynamic compression solicitation, being simultaneously mechanically stable in the wet state and exhibiting a storage modulus of $4.21 \pm 1.04 \mathrm{MPa}$ at $1 \mathrm{~Hz}$ frequency. Furthermore, chitosan scaffolds were evaluated in vivo using a rat muscle-pockets model for different implantation periods (1, 2 and 12 weeks). The histological and immunohistochemistry results have demonstrated that chitosan scaffolds can provide the required in vivo functionality. In addition, the scaffolds interconnectivity has been shown to be favourable to the connective tissues ingrowth into the scaffolds and to promote the neo-vascularization even in early stages of implantation. It is concluded that the proposed chitosan scaffolds produced by particle aggregation method are suitable alternatives, being simultaneously mechanical stable and in vivo biofunctional that might be used in load-bearing tissue engineering applications, including bone and cartilage regeneration.
\end{abstract}

(c) 2008 Elsevier Ltd. All rights reserved.

\section{Introduction}

In tissue engineering, scaffolds are typically needed, both as carriers for cells or biochemical factors, or as constructs providing appropriate mechanical conditions. In general, the term scaffold is used to describe all structures that are used to restore functionality of an organ either permanently or temporarily [1]. An ideal scaffold should have several characteristics as it has been comprehensively reviewed in several papers [1,2], such as: (i) three-dimensional (3D) and porous supports with an interconnected pore network for cell growth and flow transport of nutrients and metabolic waste with suitable surface chemistry for cell attachment, proliferation, and differentiation; (ii) mechanical properties adequate to the tissues at

\footnotetext{
* Corresponding author. 3B's Research Group - Biomaterials, Biomimetics and Biodegradables, Department of Polymer Engineering, University of Minho, Campus de Gualtar, 4710-057 Braga, Portugal. Fax: +351 253604498.

E-mail address: pmalafaya@dep.uminho.pt (P.B. Malafaya).
}

the site of implantation and (iii) biocompatibility and biodegradability with a controllable degradation and resorption rate to match cell/tissue growth in vitro and in vivo.

When considering the main aimed applications, it is not surprising that there is a wide range of scaffolds, including porous scaffolds that allow for cell adhesion, and provide biological functions $[1,3]$. In fact, from a mechanical point of view, scaffolds vary widely from soft gels, mainly serving as carriers for cells $[4,5]$ to stiff biodegradable calcium phosphate scaffolds [6,7]. Another criterion to be fulfilled is the biocompatibility or in vivo biofunctionality. All implant materials must be non-toxic to the body and prove to interact properly with the host tissue.

Another quite important requirement is that the scaffold must act as a three-dimensional (3D) template for in vitro and in vivo tissue growth, i.e., the scaffold must consist of an interconnected macroporous network allowing for cell/tissue growth and flow transport of nutrients. In the case of bone tissue engineering, it was shown $[8,9]$ that the interconnections for bone ingrowth larger than $50 \mu \mathrm{m}$ were favourable for mineralized bone formation. The 
interconnectivity of the pores dominates the flow properties, which are important to ensure adequate delivery of cells during seeding and nutrients during subsequent culture/implantation. Several investigators $[6,8,10,11]$ have also studied bone ingrowth into porous material with different pore sizes and the consensus seems to be that the optimal pore size for bone ingrowth is $100-400 \mu \mathrm{m}$ $[6,8,10,11]$. Therefore, an ideal scaffold would have interconnects of at least $50 \mu \mathrm{m}$ in diameter between its macropores of $100-400 \mu \mathrm{m}$ pore size range. It is thus important to be able to quantify both the pores and interconnects to optimize tissue scaffolds.

Effective scaffold assessment techniques are then required right at the initial stages of research and development to select or design scaffolds with suitable properties. Micro-Computed Tomography (micro-CT) appears to be such a measurement technique. Micro-CT was identified as having various key advantages over other techniques, such as its non-destructiveness and the possibility to assess many different morphometric parameters [10-12]. This technique was first proposed to analyze trabecular bone samples [13] and, since then, has been used extensively in the study of trabecular architecture, [14] and their applications in other areas are clearly increasing, namely in the tissue engineering field. The increasing use of this technique in tissue engineering [10-12] can be attributed to micro-CT capacity to provide for accurate quantitative and qualitative information on the 3D morphology of the sample. Another main advantage is that the interior of the object can be studied with great detail without physical sectioning or use of toxic chemicals. Moreover, after scanning, the integral samples can be subjected to other tests due to its non-destructive nature, thereby resolving the problem of sample scarcity. This is a very important characteristic allowing, for instance, the accurate study of bone ingrowth which is being widely used by the group of Cancedda $[6,15]$. The morphometric parameters for architectural analyses of scaffolds can be easily extrapolated based on the histomorphometric/structural indices usually measured for bone samples, such as bone surface (BS) and volume (BV), trabecular thickness (Tb. Th) and trabecular separation ( $\mathrm{Tb}$. Sp), structural degree of anisotropy (DA) or bone surface-to-volume ratio (BS/BV) [16].

Different materials have been proposed to be used as both 3D porous scaffolds and hydrogel matrices for distinct tissue engineering strategies. It is often beneficial for the scaffolds to mimic certain advantageous characteristics of the natural extracellular matrix, or developmental or wound healing cascades [17]. Ideally, scaffolds would be made of biodegradable polymers whose properties closely resemble those of the extracellular matrix (ECM), a soft, tough, and elastomeric protein-rich network, that provides mechanical stability and structural integrity to tissues and organs [18]. Chitosan appears to be an excellent alternative due its interesting and versatile properties $[19,20]$. Studies on chitosan as a potential candidate for tissue engineering scaffolding demonstrate this, as those have been clearly intensified during the past years $[19,20]$. Chitosan is a natural polymer obtained from renewable resources, obtained from the shell of shellfish, and the wastes of seafood industry. It has attractive properties such as biocompatibility, biodegradability, antibacterial, and wound healing activity [20]. Furthermore, recent studies suggested that chitosan and its derivatives are promising candidates to be used as supporting materials for tissue engineering applications, owing to their porous structure, gel forming properties, ease of chemical modification, high affinity to in vivo macromolecules, and so on [19].

Bearing all these considerations in mind, the aim of the present study was to evaluate the performance of chitosan-based scaffolds produced by a particle aggregation method proposed for tissue engineering applications. Those systems should try to meet the three key requirements for an ideal design: a porous structure with adequate morphological characteristics, adequate mechanical behaviour and an in vivo biocompatibility. To evaluate these properties, micro-Computed Tomography (micro-CT) was carried out for accurate morphometric characterization and Dynamical Mechanical Analysis (DMA) was performed to study the scaffolds behaviour in wet state under compression solicitation. Chitosan scaffolds were also evaluated in vivo using a rat muscle-pockets model for different implantation periods (1, 2 and 12 weeks) with subsequent characterization, including histological and immunohistochemical evaluation.

\section{Materials and methods}

\subsection{Scaffolds production}

The chitosan particle aggregated scaffolds were produced as described elsewhere [21]. Briefly, chitosan (medium molecular weight and deacetylation degree $\approx 85 \%$ ) was grinded and dissolved overnight in acetic acid ( $1 \% \mathrm{vv}$ ) to obtain a chitosan solution ( $2 \% \mathrm{wt}$ ). Unless otherwise stated, all chemicals were bought from Sigma-Aldrich and used as received. After complete dissolution and filtration, the prepared solutions were extruded through a syringe at a constant rate $(10 \mathrm{ml} / \mathrm{h})$ to form chitosan droplets into a $\mathrm{NaOH}(1 \mathrm{M})$ precipitation bath where particles with regular diameter were formed. The residence time of the chitosan droplets in the precipitation medium was minimized till the precipitation of chitosan particles. The same residence time was further used to produce the chitosan particles and was approximately $5 \mathrm{~min}$. The chitosan particles were collected and washed repeatedly with distilled water until neutral $\mathrm{pH}$ was reached. The particles were subsequently placed into cylindrical moulds and left to dry at $60{ }^{\circ} \mathrm{C}$ for 3 days. No significant compression force was applied other than to fit the particles into the mould adequately. The drying temperature was optimized in order to accelerate the solvent evaporation in a reasonable time period. Cylindrical shaped scaffolds with $8 \mathrm{~mm}$ height and $5 \mathrm{~mm}$ diameter were obtained. For the in vivo studies, cylindrical shaped scaffolds with $3 \mathrm{~mm}$ height and $5 \mathrm{~mm}$ diameter were used. Cross-sections of $10 \mu \mathrm{m}$ were also obtained from the scaffolds' bulk and stained with eosin for visual evaluation of the interface between the particles.

\subsection{Micro-Computed Tomography (micro-CT)}

Chitosan particle aggregated scaffolds $(n=3)$ were scanned using micro-Computed Tomography (micro-CT) for morphological and morphometric characterization. Micro-CT was carried out with a high-resolution micro-CT SkyScan 1072 scanner (Skyscan, Kontich, Belgium) using a resolution of pixel size of $8.79 \mu \mathrm{m}$ and integration time of $1.9 \mathrm{~ms}$. The X-ray source was set at $70 \mathrm{keV}$ of energy and $145 \mu \mathrm{A}$ of current. Approximately 400 projections were acquired over a rotation range of $180^{\circ}$ with a rotation step of $0.45^{\circ}$. Data sets were reconstructed using standardized cone-beam reconstruction software (NRecon v1.4.3, SkyScan). The output format for each sample was 850 serial $1024 \times 1024$ bitmap images. Representative data sets of 200 slices were segmented into binary images with a dynamic threshold of 60-255 (grey values). This data was used for morphometric analysis (CT Analyser v1.5.1.5, SkyScan) and to build 3D virtual models (ANT 3D creator v2.4, SkyScan). The morphometric analysis included porosity, scaffolds interconnectivity, mean pore and particle size and respective distribution. 3D virtual models of representative regions in the bulk of the scaffolds were created, visualized and registered using both image processing softwares CT Analyser and ANT 3D creator. In order to characterize the porosity morphology of the scaffold, threshold inversion was used to create a $3 \mathrm{D}$ model.

\subsection{Dynamic Mechanical Analysis (DMA)}

Dynamic Mechanical Analysis (DMA) was conducted in order to characterize the dynamic mechanical behaviour of chitosan particle aggregated scaffolds in wet state under dynamic compression solicitation. Chitosan cylindrical scaffolds were immersed in Phosphate Buffer Solution (PBS) at physiological $\mathrm{pH}$ for 3 days for complete hydration. The scaffolds were then subjected to compression cycles of increasing frequencies ranging from 0.1 to $40 \mathrm{~Hz}$ with constant amplitude displacements of $0.03 \mathrm{~mm}$ using a Tritec 2000 DMA (Triton Technology, UK). Experiments were conducted at room temperature and five points were measured within each decade. For each individual scaffold, the data is averaged over three consecutive runs. Five samples were measured for each type of scaffolds. The real (storage modulus), $E^{\prime}$, and the imaginary component (loss modulus), $E^{\prime \prime}$, of the complex modulus, $E^{*}=E^{\prime}+i E^{\prime \prime}$ (with $i=(-1)^{1 / 2}$ ), were recorded against frequency. Reference values for the compression modulus were collected at a frequency of $1 \mathrm{~Hz}$.

\subsection{In vivo biocompatibility study}

\subsubsection{Surgical procedure}

Four male Sprague Dawley rats, weighing 350-380 g were used for intramuscular implantation of the chitosan aggregated scaffolds. Each animal was anaesthetized (induction with $3-3.5 \%$ isofluorane and $7 \mathrm{~L} / \mathrm{min}$ of air for $2-3 \mathrm{~min}$; maintenance with intramuscular injection of $90 \mathrm{mg} / \mathrm{kg}$ of ketamin combined with 
Table 1

Primary antibodies used in the immunohistochemistry evaluation of the explants

\begin{tabular}{|c|c|c|c|c|c|}
\hline Antibody & Recognizes & Dilution & $\begin{array}{l}\text { Incubation/ } \\
\text { temperature }\end{array}$ & Company & Country \\
\hline$\overline{\mathrm{CD} 18}$ & $\begin{array}{l}\text { Rat } \beta 2 \text { integrins } \\
\text { ( } \beta \text { chain of LFA- } 1 \text { ) }\end{array}$ & $1: 100$ & $1 \mathrm{~h} / \mathrm{RT}$ & Serotec & UK \\
\hline CD3 & T lymphocytes & $1: 30$ & $1 \mathrm{~h} / \mathrm{RT}$ & DakoCytom & Denmark \\
\hline vWF & von Willebrand factor & $1: 200$ & $1 \mathrm{~h} / \mathrm{RT}$ & DakoCytomation & Denmark \\
\hline SMA & Smooth muscle actin & $1: 5000$ & $1 \mathrm{~h} / \mathrm{RT}$ & Sigma & USA \\
\hline
\end{tabular}

$5 \mathrm{mg} / \mathrm{kg}$ xylazine) and subjected to local tricotomy for adequate surgical procedure. The skin was disinfected and under surgical sterile conditions, four lumbar paravertebral incisions of approximately $2 \mathrm{~cm}$ length were performed through the cutis, subcutis and the panniculus carnosus (smooth skin muscle). After incising the fascia of the latissimus dorsi muscle, craniolateral oriented muscle-pockets were created by blunt dissection. Into these pockets, the scaffolds were inserted and the fascia carefully sutured, as well as the panniculus carnosus and finally the skin. As an analgesic treatment, animals received Metamizol $(200 \mu \mathrm{g} / \mathrm{g}$ of body weight per day in drinking water ad libitum). At 1,2 and 12 weeks post-implantation, the animals were anaesthetized (see above) and sacrificed by an intracardial overdose of pentobarbital. The scaffolds and surrounding tissue were explanted for further evaluation. The animal experiment was approved by the local ethical authorities responsible for animal experimentation.

\subsubsection{Explants morphological characterization}

At 1,2 and 12 weeks post-implantation, explants were registered using stereolight microscopy. Furthermore, micro-Computed Tomography (micro-CT) of the explants was carried out as described previously. Two-dimensional X-ray photographs were registered from the bulk of the explants.

\subsubsection{Histological evaluation}

After explantation at 1,2 and 12 weeks, samples were fixed in $4.7 \%$ formalin for $24 \mathrm{~h}$ and then kept in 70\% ethanol. After fixation, samples were dehydrated in graded ethanol (50, 70, 95 and 100\%), cleared in xylene and then embedded in paraffin. Afterwards, samples were sectioned in 2-4 $\mu \mathrm{m}$ sections and stained with haematoxylin/eosin (H\&E). The stained microscope slides were observed by, at least, two independent observers.

2.4.3.1. Immunohistochemistry. In order to perform immunohistochemistry, paraffin was removed from the samples slides upon heating. They were hydrated in descending ethanol concentrations (100, 95, 70 and 50\%) and incubated in phosphate buffer saline (PBS). The antigen retrieval was performed with microwave incubation in citrate buffer $(\mathrm{pH}=6)$ at $800 \mathrm{~W}$ for $20 \mathrm{~min}$ and the slides were washed with PBS. The endogenous peroxidase was blocked with $1 \%$ hydrogen peroxide $\left(\mathrm{H}_{2} \mathrm{O}_{2}\right)$ in methanol, at room temperature (RT) for $15 \mathrm{~min}$. After washing, the slides were incubated with the primary antibodies at RT for $1 \mathrm{~h}$ (see Table 1 ) and washed at the end of the incubation period. Subsequently, the samples were incubated with the secondary antibody (Polyclonal Swine Anti-Goat, Mouse, Rabbit Immunoglobulins/Biotinylated - DakoCytomation, Denmark; dilution 1:100) at RT, for $30 \mathrm{~min}$. After washing with PBS, the samples were incubated with the ABComplex (StreptABComplex/HRP, DakoCytomation, Denmark) at RT for $30 \mathrm{~min}$ and washed again after the incubation period. Visualisation of the bound antibodies in the samples was revealed by incubation with AEC substrate (Dako RealTM EnVisionTM Detection System, Peroxidase/DAB+, Rabbit/Mouse; DakoCytomation, Denmark) until the colour could be detected. The revelation of the labelling was stopped with PBS and the samples were stained with Mayer's Haematoxylin for nuclear contrast, at RT for $2 \mathrm{~min}$. After this, the samples were washed with distilled water, dehydrated in graded ethanol (50, 70, 95 and 100\%), cleared with xylene substitute, permanently mounted with Clarion ${ }^{\mathrm{TM}}$ Mounting Medium, and observed in the light microscope by, at least, two independent observers.

\section{Results}

\subsection{Morphometric analysis}

With the emerging of the advances on computer technology, micro-CT has become a powerful tool for analyzing porous materials based on 3D geometrical considerations. Micro-CT can provide virtual representations and accurate morphometric parameters of structural characteristics in a non-destructive and reproducible way that generates precise three-dimensional (3D) measurements of scaffold architecture [11,12]. One of the key parameters of microCT that one has to define is the threshold that is the basis for an adequate morphometric analysis. In Fig. 1, we represent a characteristic histogram for chitosan particle aggregated scaffolds that allows us to define the dynamic threshold used for further morphometric analysis and 3D models generation.

Quantitative analysis of the scaffolds architecture such as porosity or pore size distribution can be obtained by micro-CT based on the structural indexes usually measured for bone samples [14]. Scaffold (bone) volume (BV) is calculated using tetrahedrons corresponding to the enclosed volume of the triangulated surface [16]. Total volume (TV) is the overall volume of the scanned scaffold. Scaffolds porosity (\%) can, therefore, be calculated as (1-BV/TV). Particle size (trabecular thickness) or pore diameter (trabecular separation) as well as their distribution can also be computed. Furthermore, interconnectivity can also be calculated using an appropriate pore size limit. The referred values can be computed as an average of all the $2 \mathrm{D}$ measurements giving information of the

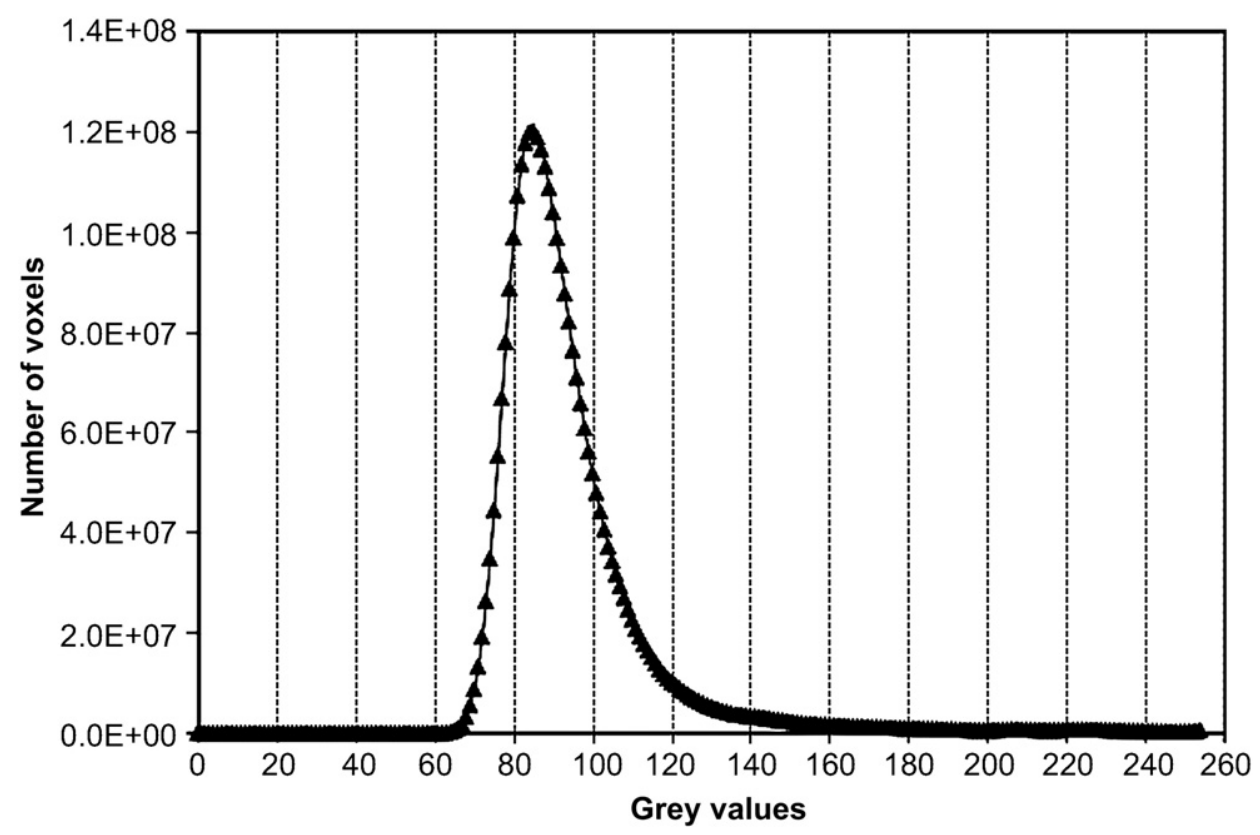

Fig. 1. Representative histogram obtained for chitosan particle aggregated scaffolds used to define the dynamic threshold (grey values). 

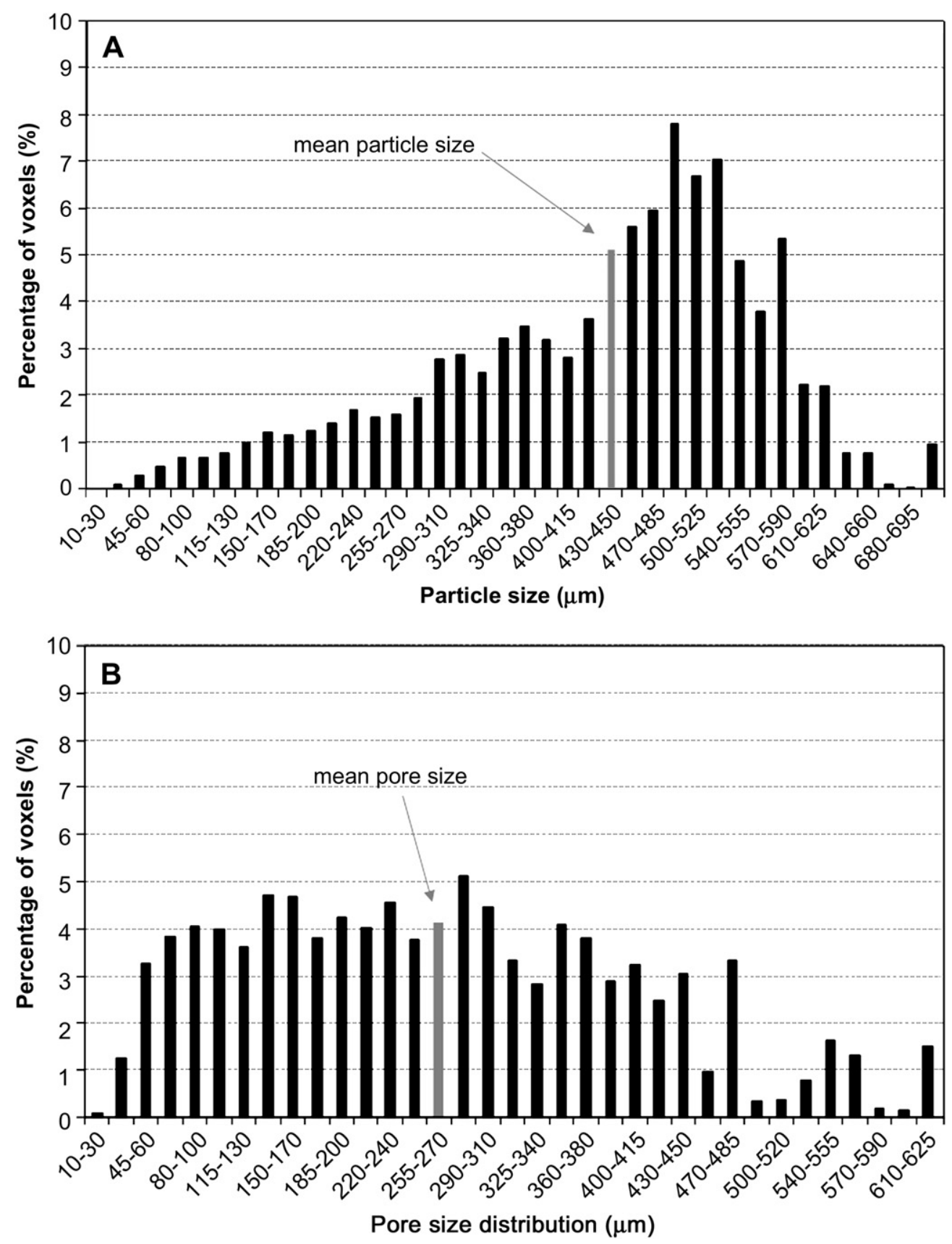

Fig. 2. Particle (A) and pore (B) size distribution of chitosan particle aggregated scaffolds assessed by 3D micro-CT analysis.

parameters behaviour along the scaffolds (such as porosity distribution) or more accurately in $3 \mathrm{D}$ analysis, as performed in the present paper.

Porosity and interconnectivity of the chitosan particle aggregated scaffolds were also computed. Percentage values of $27.78 \pm 2.80 \%$ and $94.99 \pm 1.41 \%$ (mean \pm SD) were found for both parameters, respectively. It is important to stress out that interconnectivity was calculated with a limit in pore size of $53 \mu \mathrm{m}$ as minimum value for interconnected pores, meaning that interconnection diameters lower than this value were consider as closed pores.

The average particle size was $430.72 \pm 54.42 \mu \mathrm{m}$ and the average pore diameter was $265.46 \pm 24.27 \mu \mathrm{m}$ and their distribution is disclosed in Fig. 2. By analysing the pore size distribution, one can observe that the chitosan-based scaffolds present a heterogeneous range of pore size covering the optimal pore size reported as consensus for tissue engineering applications $[6,8,10,11]$.

\subsection{Dynamic Mechanical Analysis (DMA)}

As previously discussed, one of the key issues of scaffolds design for tissue engineering is their mechanical performance. Therefore, the assessment of scaffolds' mechanical behaviour is essential to ensure the mechanical stability, namely in hydrated state approaching the in vivo condition where physiological fluids are present. In the present paper, chitosan particle aggregated scaffolds were characterized in the wet state over a range of physiological frequency range in load-bearing applications, such as articular cartilage or bone $[22,23]$. The reference values at a frequency of $1 \mathrm{~Hz}$ for elastic (storage) and viscous (loss) components of the complex modulus were found to be $4.21 \pm 1.04 \mathrm{MPa}$ and $0.36 \pm 0.07 \mathrm{MPa}$ (mean $\pm \mathrm{SD}$ ), respectively. The storage ( $\left.E^{\prime}\right)$ and loss $\left(E^{\prime \prime}\right)$ modulus behaviour for increasing frequencies is presented in Fig. 3 and the frequency dependence of the loss factor $(\tan \delta)$ is 


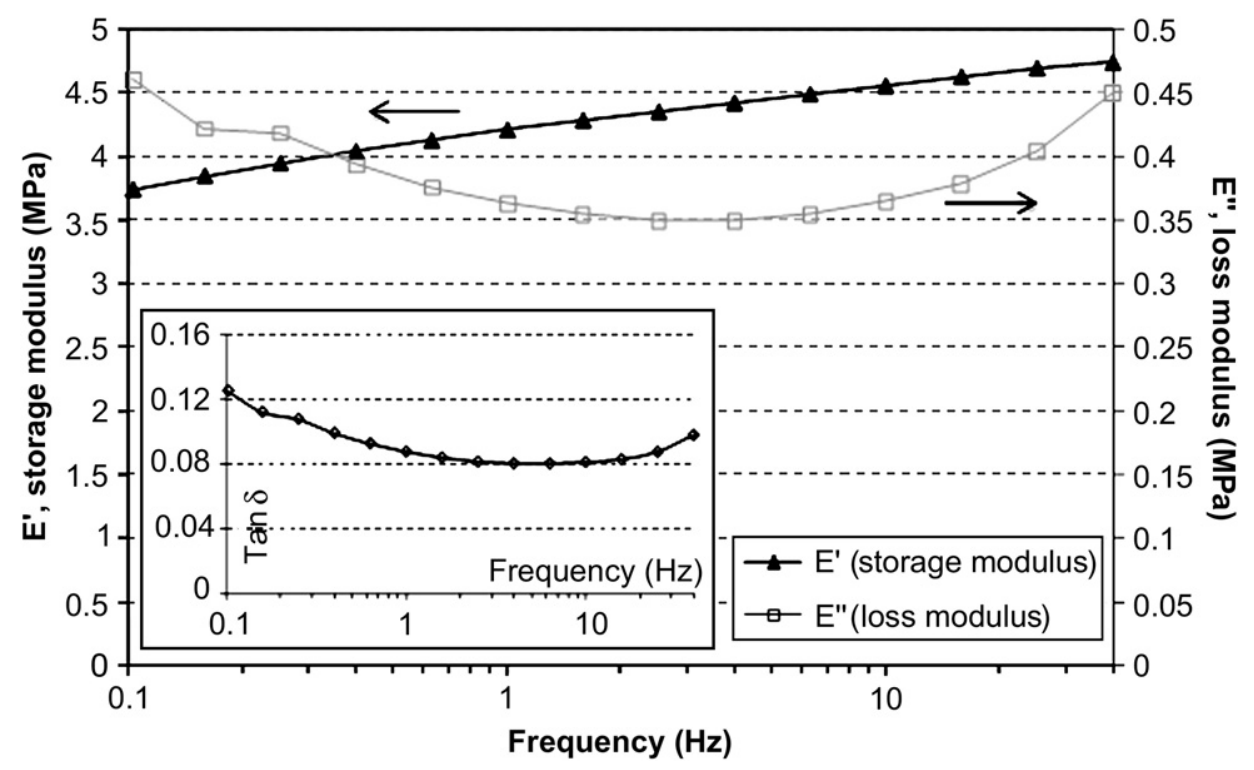

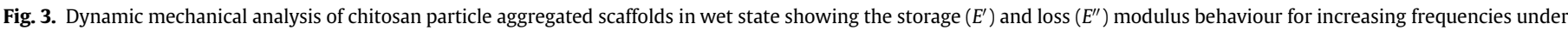
dynamic compression solicitation. The inset graph shows the frequency dependence of the loss factor $(\tan \delta)$.

shown in the inset graph. As it is possible to observe, the elastic modulus $\left(E^{\prime}\right)$ increases with increasing frequency, being typically above $4 \mathrm{MPa}$, while the viscous modulus $\left(E^{\prime \prime}\right)$ shows the inverse trend with a slight increase for higher frequencies. As a consequence, there is an obvious decrease of the loss factor, ( $\tan \delta=E^{\prime \prime}$ | $E^{\prime}$ ) with increasing frequency with a slight increase for higher frequencies as shown in the inset graph.

\subsection{In vivo biocompatibility}

No systemic or regional surgical complications were seen for any of the rats in the post-operative period. A morphological characterization of the explants, after the different implantation periods, showed connective tissue ingrowth into the chitosan scaffolds even after 1 week of implantation (Fig. 4). This tissue ingrowth becomes more pronounced with increasing time periods of implantation. This was further confirmed by micro-CT. The 2D
X-Ray evaluation of the cross-sections in the explants' bulk is presented in Fig. 5 where it is possible to identify the increase of the host tissue inside the scaffolds pores with the implantation time. Furthermore, there is a macroscopic indication of vascularization of the implants after 12 weeks of implantation (Fig. 4E and F).

Histology, including immunohistochemistry analysis, was performed after the different implantation periods, and the obtained results are disclosed in the following Figs. 6-10. After 1-week implantation of the chitosan particle aggregated scaffolds, the predominant inflammatory cell type found was the polymorphonuclear neutrophil (PMNs) recruited from the circulation in response to the implanted material (Fig. 6A). Some other inflammatory cells, such as lymphocytes and macrophages can also be observed, but in a less extent compared with the PMNs. In addition, the recruited PMNs were labelled by anti-CD18, which has affinity with the integrins, binding to the surface of recruited leukocytes (Fig. 7B). The lymphocytes present in the histological samples were labelled by
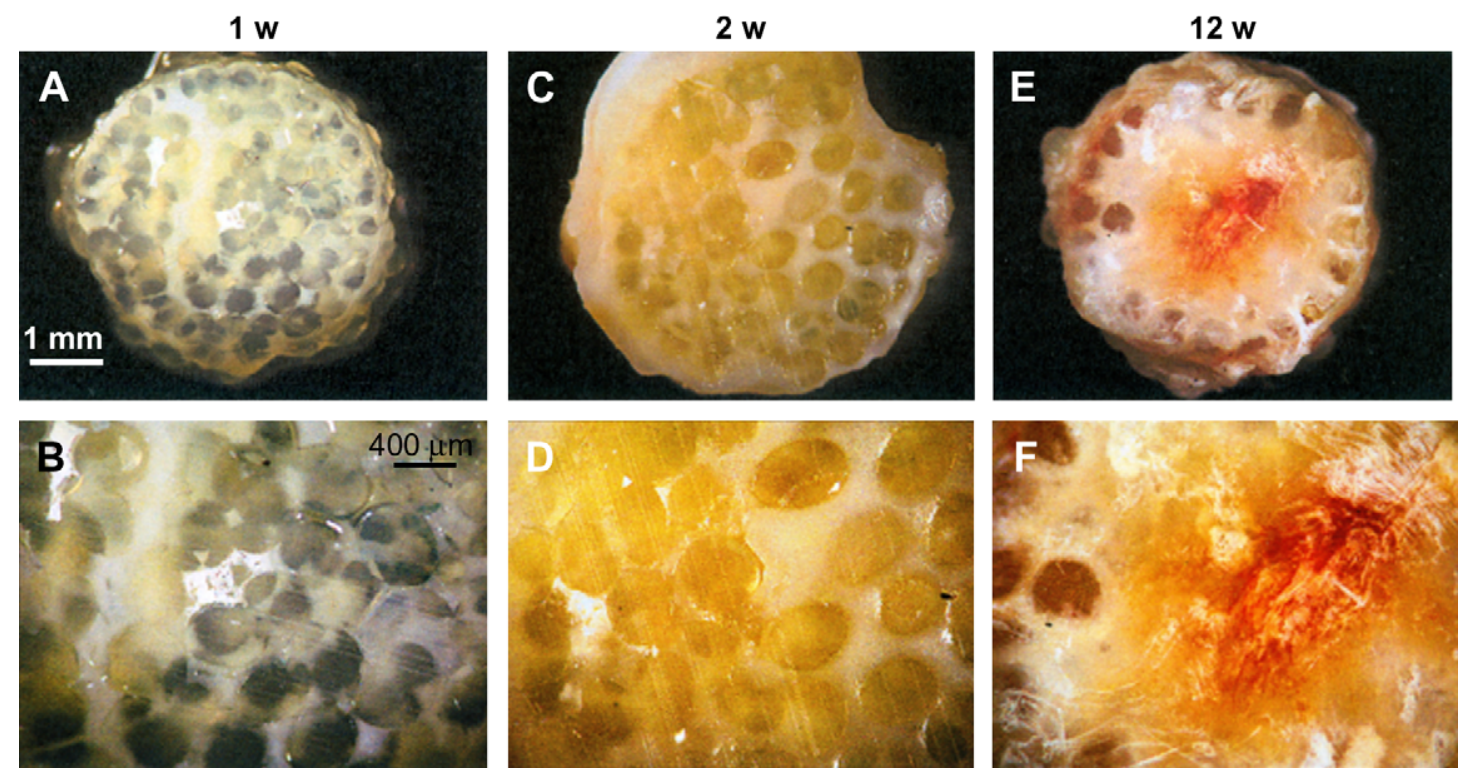

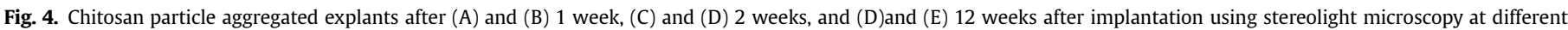
magnifications. 


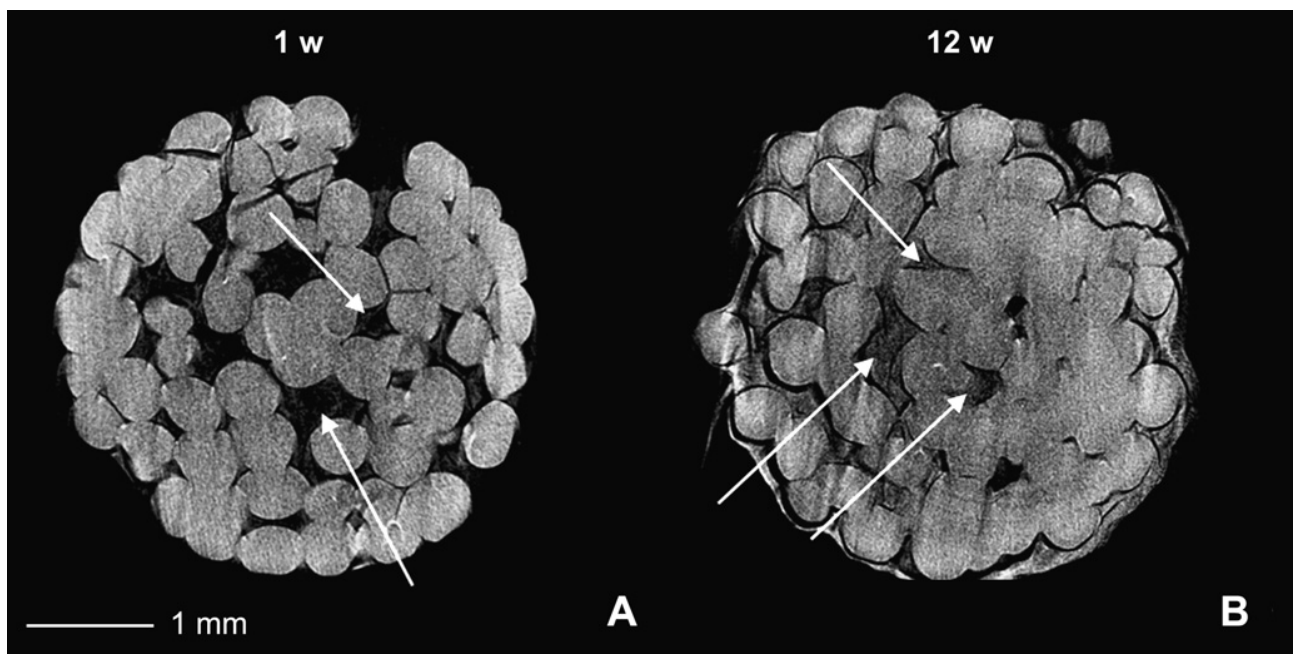

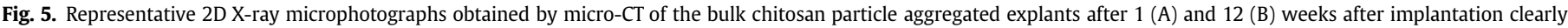
showing the tissue ingrowth indicated by the arrows.

anti-CD3 (specific for the T lymphocytes subset) and shown to be residual when compared to PMNs (Fig. 8B). Few new blood vessels could be found at this stage (Figs. 9B and 10B), in spite of the expression of $v W F$ at this time of implantation which is a common endothelial marker during the angiogenic process [24]. In addition, it is possible to identify a rudimentary extracellular matrix produced in the pores of the chitosan particle aggregated scaffolds, although it is still much disorganized (Figs. 6A and 10B).

After 2 weeks of implantation, the main inflammatory cells present were shown to be lymphocytes (Fig. 6B), which was confirmed with their specific labelling (Fig. 8C). Some remaining PMNs could still be observed (Fig. 7C), as well as some foreign body giant cells, resulting from the fusion of macrophages trying to phagocyte the implant (Fig. 6.B). Although some necrosis can be observed in the periphery of the implant (Fig. 6B), it can be neglected if compared with the amount of extracellular matrix formed around the particles of the scaffold (Figs. 6B and 10C), which shows a more organized structure compared with 1 week of implantation. Furthermore, the presence of smooth muscle actin is more evident (Fig. 10C), and allows to say that, the connective tissue is growing and the neo-vascularization is also increasing between the particles of the scaffold. This fact is further supported by the expression of vWF at this implantation period (Fig. 9C).

Three months after the implantation of the chitosan particle aggregated scaffolds, some of the remaining PMNs showed to be apoptotic (Fig. 6C) and the spaces between the particle of the scaffolds are, in this stage, filled with connective tissue cells (Fig. 6C). It was possible to observe that the PMNs appeared in much less amount compared with the other implantation times (Fig. 7D). Some lymphocytes are, as well, still present in the infiltrate (Fig. 8D). Furthermore, the general expression of vWF observed at 1 week of implantation due to the initially formed clot is now less evident, being much more restricted to the vascularized tissue (Fig. 9D). At this stage of implantation it is clear that the extracellular matrix shows to be much more organized along and between the particles of the scaffold (Figs. 6C and 10D). Furthermore, the neo-vascularization already observed in earlier times of implantation increased as the SMA labelling shows in the newly formed blood vessels (Fig. 10D). These features demonstrate that the scaffolds were well tolerated by the host and the ingrowth of host connective tissue into the scaffolds structure is an evidence of good integration.

\section{Discussion}

\subsection{Morphometric analysis}

For tissue engineering applications, it is important to ensure that the scaffold has an adequate architecture in order to allow for optimal cell migration and nutrients supply to assure optimal tissue growth in an in vivo situation. In order to be able to assess these morphometric requirements, micro-CT is becoming a powerful tool to compare scaffold production techniques [11,12]. Direct micro-CT based imaging analysis is non-destructive and non-invasive and allows precise 3D measurements of scaffold architecture quantifying scaffold porosity, surface area, and 3D measures such as
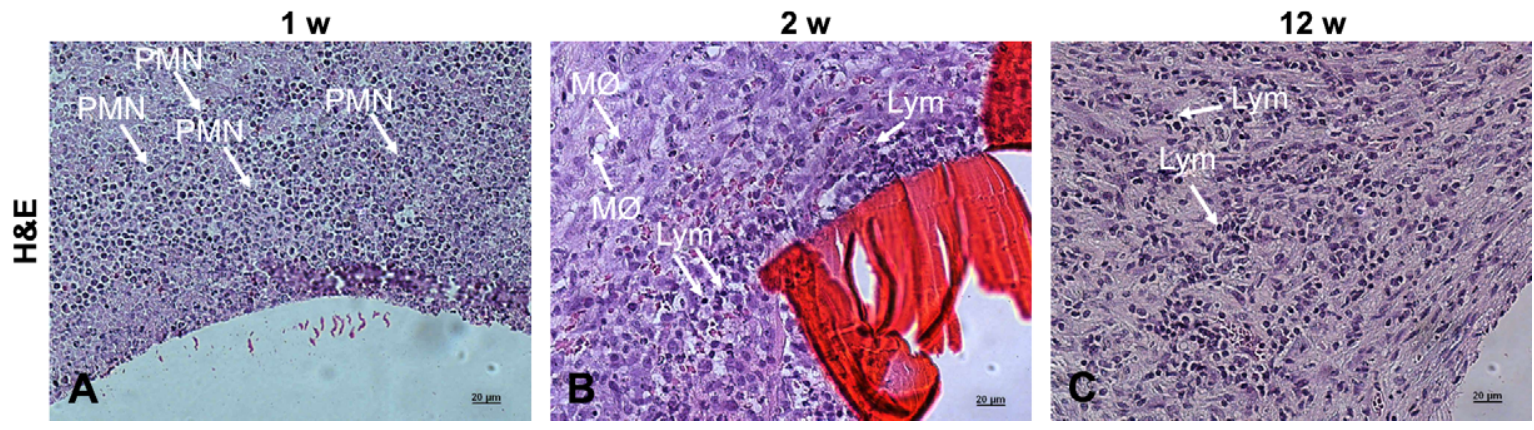

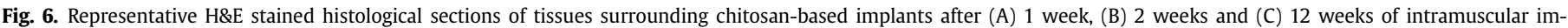
plantation. PMN - polymorphonuclear neutrophil, MØ - macrophage, and Lym - lymphocyte. Bar scale: $20 \mu \mathrm{m}$. 

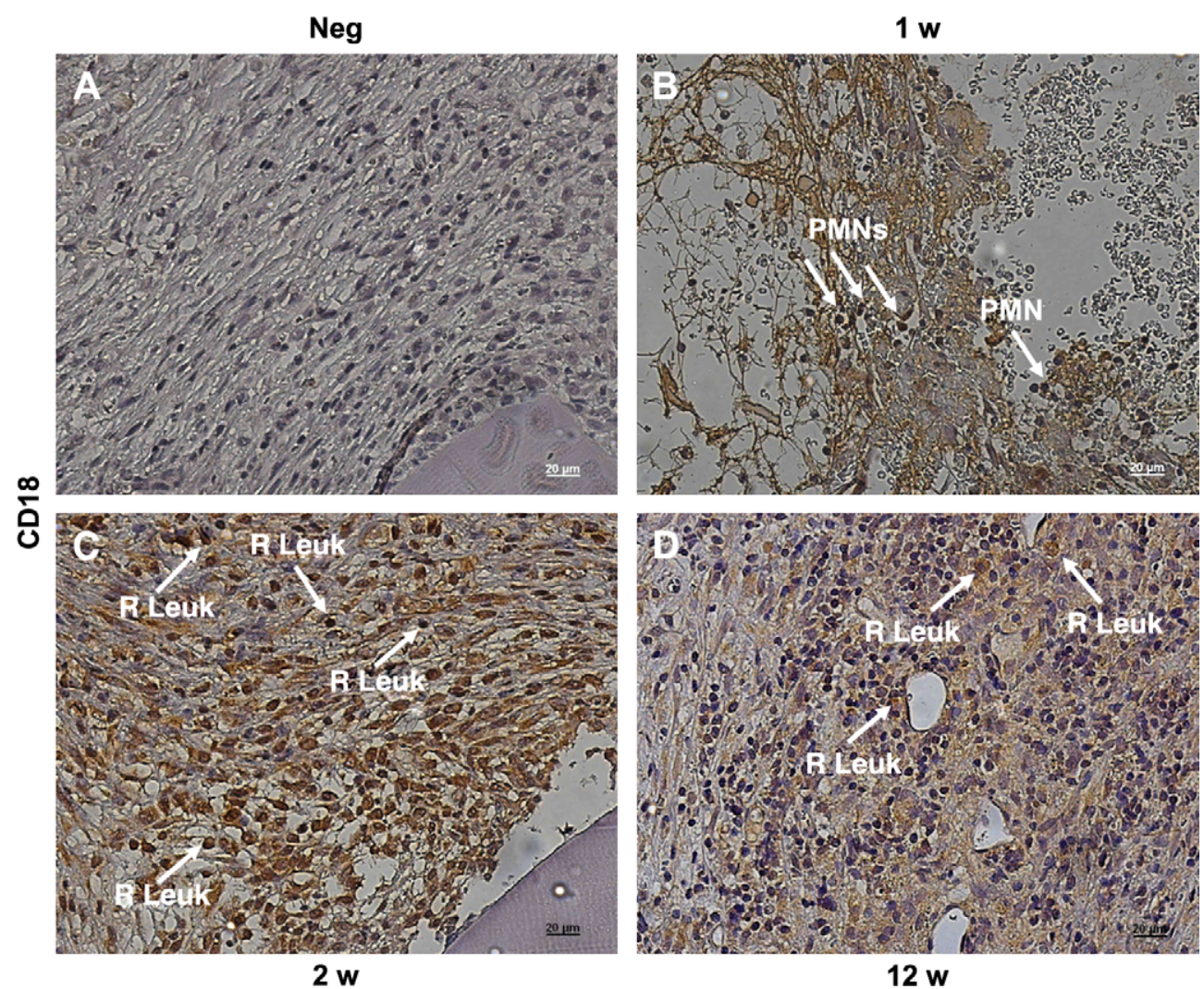

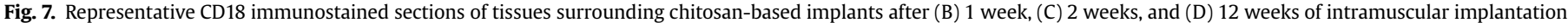
(A) Negative control. PMN - polymorphonuclear neutrophil, and R Leuk - recruited leukocytes. Bar scale: $20 \mu \mathrm{m}$.

interconnectivity, pore size and distribution, and pore wall thickness providing valuable knowledge to optimize scaffold design. These combined advantages over other morphological characterization techniques are well discussed in a review by Ho and Hutmacher [12] and, in our opinion, clearly justify why micro-CT has become a very popular technique for characterizing porous scaffolds $[12,16]$ or bone ingrowth $[10,11]$. As a result, this methodology is very useful for quality control of scaffold fabrication processes and all data obtained for computational models will be helpful in further analyses, in order to improve our understanding of mechanical and biochemical stimuli on tissue formation.

In the present work, due to the above mentioned advantages, micro-CT was used to assess the morphometric relevant parameters and $3 \mathrm{D}$ reconstructions of porous structures allowing to visualize the internal cellular structure. Particle aggregation methodology allows for the production of suitable scaffolds for load-bearing bone applications. Trabecular bone typically consists of $30 \%$ bone tissue and $70 \%$ void volume [14]. Our effort was to fabricate scaffolds by the particle aggregation that should have a total pore volume similar to the percent bone, as previously reported [21]. It is expected that as an in vivo bone defect heals, bone tissue grows into the porous system of the scaffold, and the scaffold itself degrades completely (allowing for 30\% ingrowth) to obtain healing of the bone defect. The chitosan scaffolds produced by this methodology have pore volumes of approximately $30 \%$ as discussed before. Furthermore, and using threshold inversion, micro-CT allows to visualize the pores network giving a clear image of scaffolds' porosity morphology which resemble the bone trabecular structure, as shown in Fig. 11.

One can still consider that the porosity value is low for the typical reported requirements for tissue engineering scaffolding $[1,2]$. But we have to consider that there is also the compromise between porosity and mechanical behaviour. Since the scaffolds present a high interconnectivity degree, the mechanical performance appears as more critical when aiming at load-bearing tissue engineering applications, such as bone and cartilage. Furthermore, pore size is another important factor for tissue ingrowth. Several researchers have studied bone ingrowth into porous material with different pore sizes $[6,8,10,11]$ and the consensus seems to be that the optimal pore size for bone ingrowth is $100-400 \mu \mathrm{m}[6,8,10,11]$. The proposed scaffolds have shown to present a pore size distribution in the range of the optimal pore size (Fig. 2). The 3D structures present pores with irregular shape and heterogeneous sizes (Fig. 11) because pores are created by the interstices between the particles after aggregation. Again, the pore morphology resembles those in trabecular bone where trabaculae are irregular in shape and size.

Another key issue on tissue engineering scaffolding is the interconnectivity which has direct implications for cell growth, cell migration, flow of nutrients and tissue growth into scaffolds. The proposed scaffolds are highly interconnected (Fig. 11) to assure these biological requirements. For instances, Lu et al. [9] reported the importance of interconnections for bone ingrowth and showed that interconnections larger than $50 \mu \mathrm{m}$ were favourable for mineralized bone formation. This value, thought to be the minimum pore size for good tissue ingrowth, is also been considered in the study by Otsuki et al. [10] and, for that reason, was used to calculate the interconnectivity values in the present study, meaning that the interconnectivity only considers pore interconnections higher than $53 \mu \mathrm{m}$. If the pore interconnection is lower than $53 \mu \mathrm{m}$ it is considered as a close pore. In general, this limit is not considered or at least is not reported in the majority of published morphometric studies and it is of major importance since it can change significantly the values of interconnectivity as shown in Table 2 . If we used a lower value in the limit of pore size, the interconnectivity clearly increase, as disclosed in Table 2. Nevertheless, the 


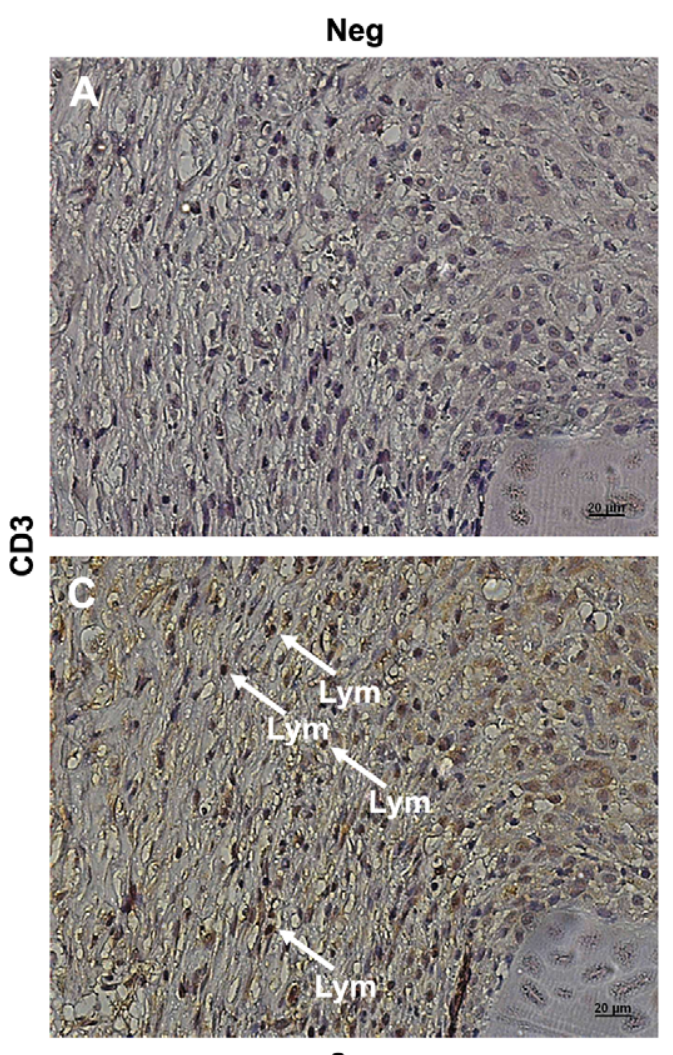

2 w
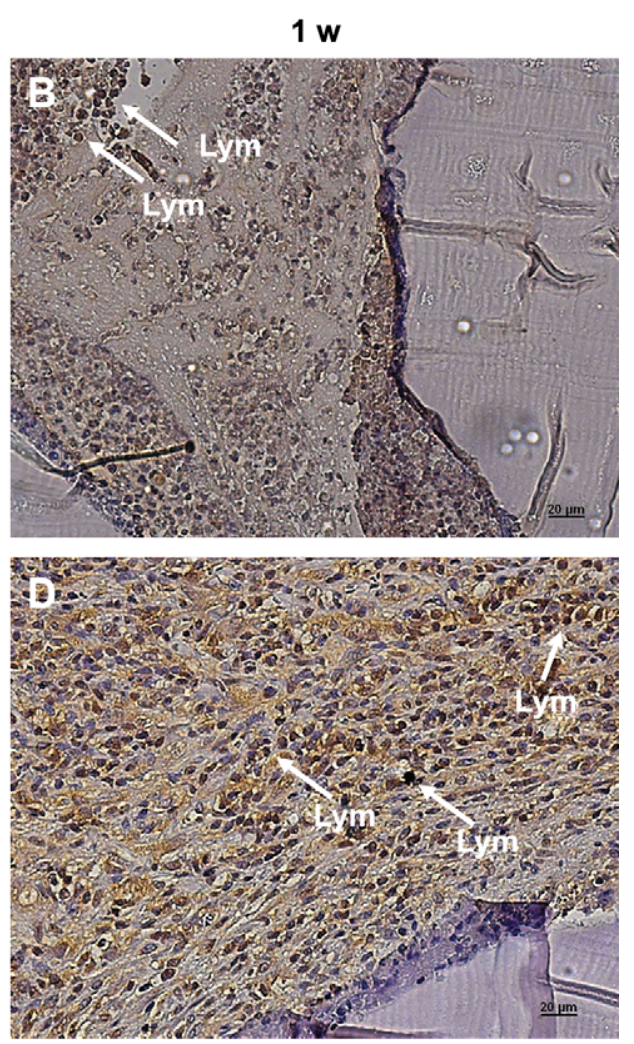

12 w

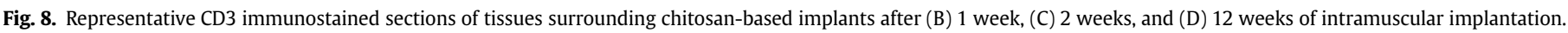
(A) Negative control. Lym - lymphocyte. Bar scale: $20 \mu \mathrm{m}$.

interconnectivity with low pore interconnection values will show no relevance when translated to in vivo situations, because the minimum pore size considered will not promote adequate tissue ingrowth. The results of the micro-CT analysis implied that the scaffolds have a suitable architecture for tissue engineering applications.

\subsection{Dynamic Mechanical Analysis (DMA)}

The maintenance of sufficient structural integrity of scaffolds is critical in tissue engineering applications, more so since the cell and tissue remodelling is important for achieving stable biomechanical conditions at the host site. Additionally, in the case of load-bearing tissue such as articular cartilage and bone, the scaffold matrix must provide sufficient temporary mechanical support to withstand in vivo stresses and loading [1,3,12]. Previous work [21] has shown that chitosan scaffolds produced under the same conditions, with the same percentage porosity as the scaffolds used in this study had a compressive modulus of $132 \pm 7 \mathrm{MPa}$ in dry state under static compression solicitation. The scaffolds showed a very good mechanical behaviour when compared to the typical mechanical properties obtained for chitosan-based porous materials even if in composite form $[25,26]$. The relatively high mechanical properties obtained by the particle aggregation methodology was further supported by other works, namely the work by Jiang et al. [27] when using chitosan microspheres in this case with poly(lactic acidglycolic acid) sintered together to obtain the final 3D structure.

Nevertheless, it is common to study the scaffolds mechanical performance in dry state and static conditions. In the present study, we have evaluated the mechanical performance of the chitosan particle aggregated scaffolds in hydrated state under dynamic solicitation, mimicking in this way the in vivo physiological condition in a post-implantation scenario. The dynamic mechanical behaviour of the scaffolds was characterized by DMA. Both storage and loss modulus ( $E^{\prime}$ and $E^{\prime \prime}$ ) were measured in the frequency range $0.1-40 \mathrm{~Hz}$, which are typical frequencies found in physiological situations in load-bearing applications [22,23]. The storage modulus $\left(E^{\prime}\right)$ is about one order of magnitude higher than the loss modulus $\left(E^{\prime \prime}\right)$ indicating an elastic nature of the chitosan scaffolds in the hydrated state. The storage modulus increases with increasing frequency $E^{\prime}$ while the loss modulus shows the inverse tendency except for high frequencies. This results in a decrease of the $\tan \delta$ (loss factor) with increasing frequency with a small increase for high frequencies (inset graphic in Fig. 3). This factor measures the proportion of the imposed mechanical stress that is dissipated in the form of heat. As $\tan \delta$ is typically above 0.08 for $f<1 \mathrm{~Hz}$, one may conclude that the scaffolds possesses significant damping capability that may be useful to dissipate some cyclic mechanical energy that is imposed in an implantation scenario. Nevertheless, there is an increase in $E^{\prime \prime}$ for high frequencies, which suggests that the material exhibits some dissipation capability for high frequencies. The developed scaffolds present elastic and viscous modulus of $4.21 \pm 1.04 \mathrm{MPa}$ and $0.36 \pm 0.07 \mathrm{MPa}$, respectively, at a frequency of $1 \mathrm{~Hz}$. The obtained compression modulus in wet state of the scaffolds produced by the particle aggregation method presents considerable higher values when compared to other chitosan-based scaffolds with fibre-based architectures produced by fibre bonding which present a higher porosity [28]. Furthermore, it is important to keep in mind that the mechanical properties may be further improved if desired with crosslinking or by incorporating a ceramic filler.

The relatively good mechanical performance of the chitosan particle aggregated scaffolds is attributed to the stable interface between the chitosan particles as shown in the 3D virtual model obtained by micro-CT (Fig. 12). The 3D virtual model shows the bonding between the adjoining chitosan particles achieved by 


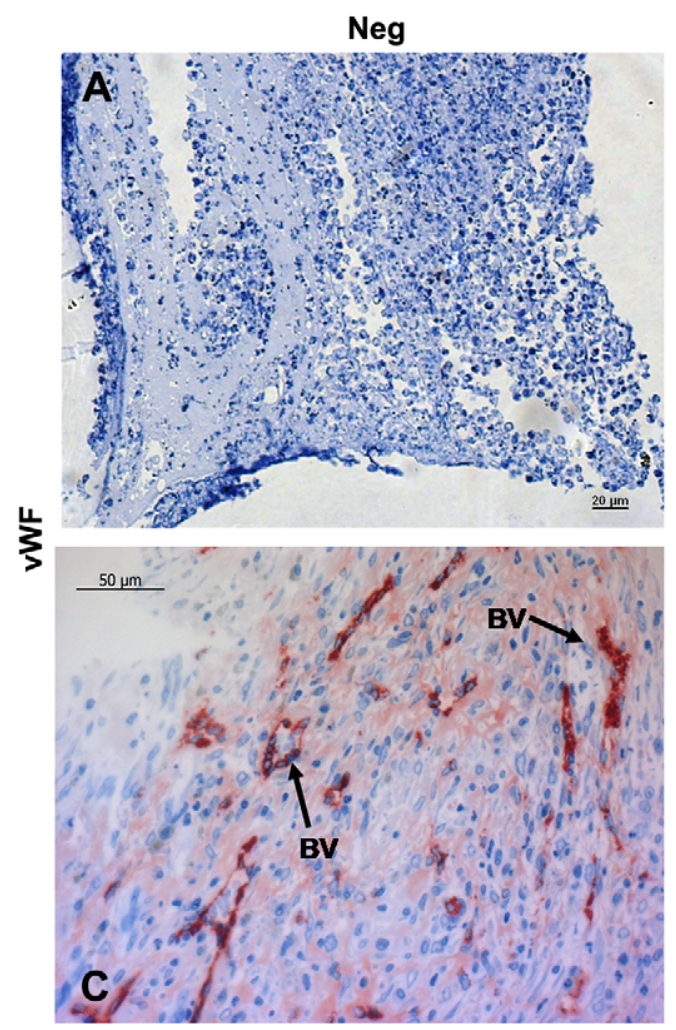

$2 \mathbf{w}$
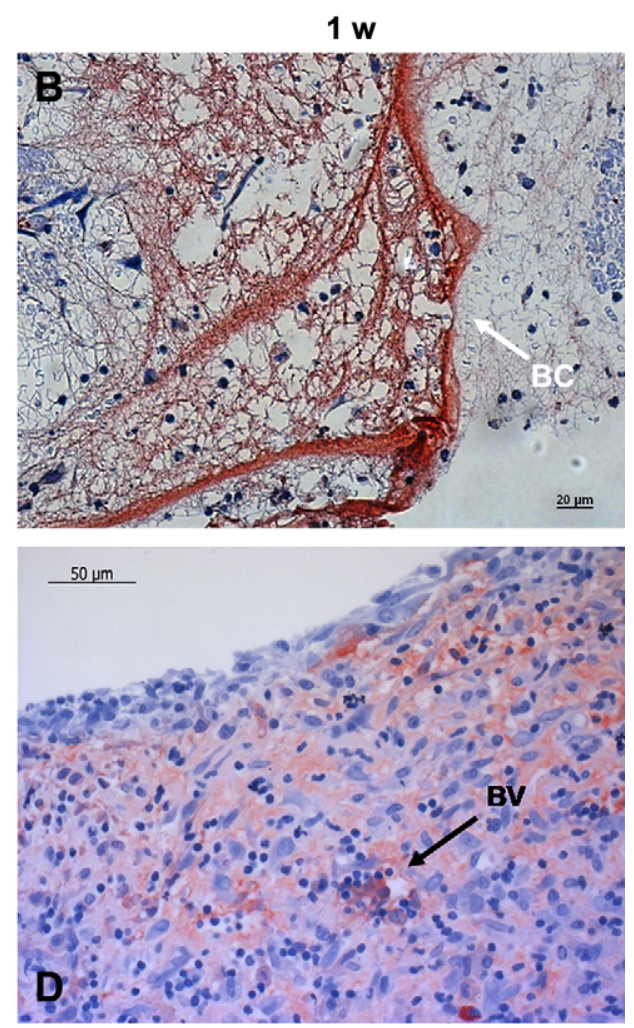

12 w

Fig. 9. Representative vWF immunostained sections of tissues surrounding chitosan-based implants after (B) 1 week, (C) 2 weeks, and (D) 12 weeks of intramuscular implantation. (A) Negative control. BC - Blood Clot, and BV - blood vessel. Bar scale: $20 \mu \mathrm{m}$ (A, B) and $50 \mu \mathrm{m}$ (C, D).
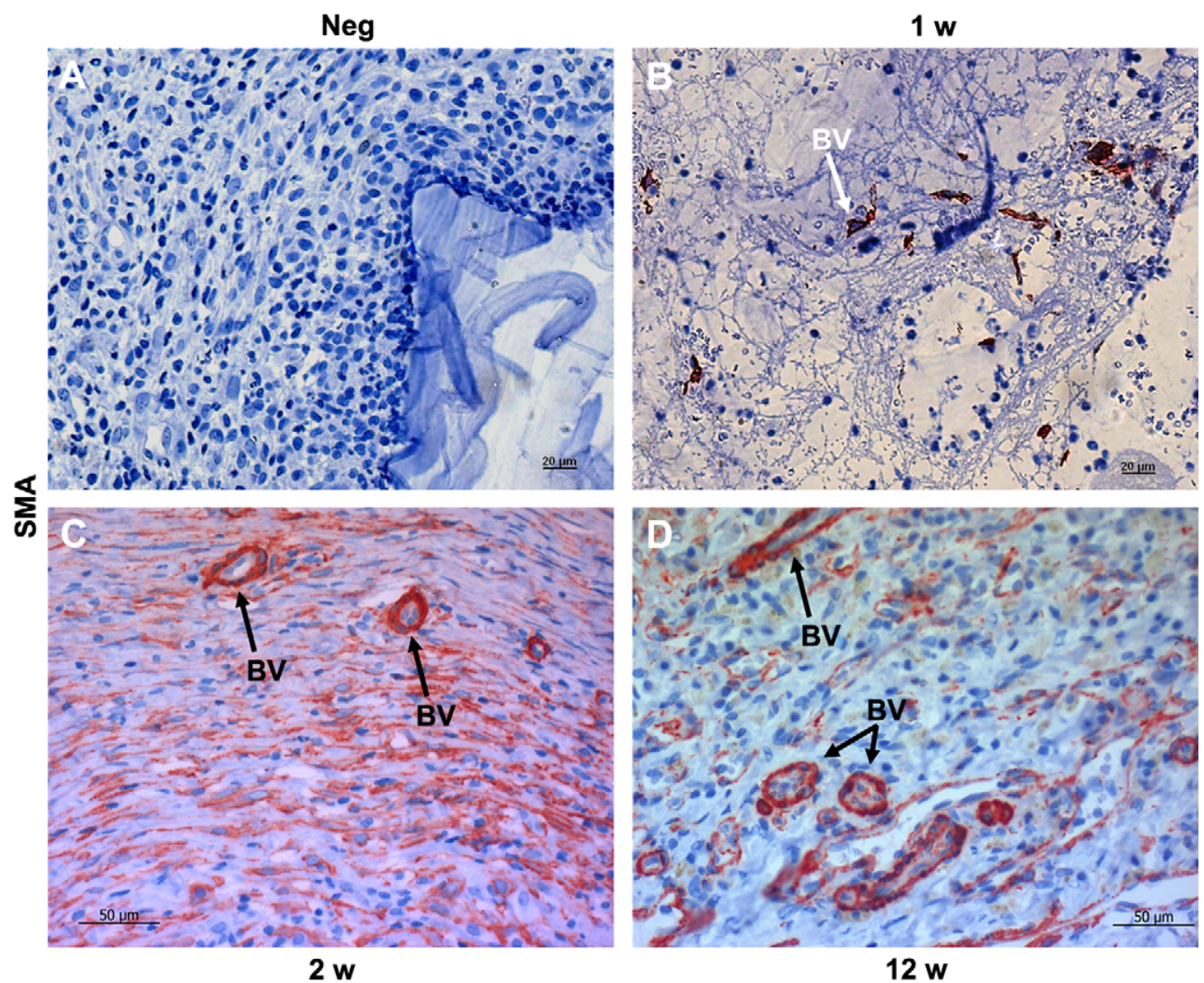

Fig. 10. Representative SMA immunostained sections of tissues surrounding chitosan-based implants after (B) 1 week, (C) 2 weeks, and (D) 12 weeks of intramuscular implantation. (A) Negative control. BV - blood vessel. Bar scale: $20 \mu \mathrm{m}$ (A, B) and $50 \mu \mathrm{m}$ (C, D). 


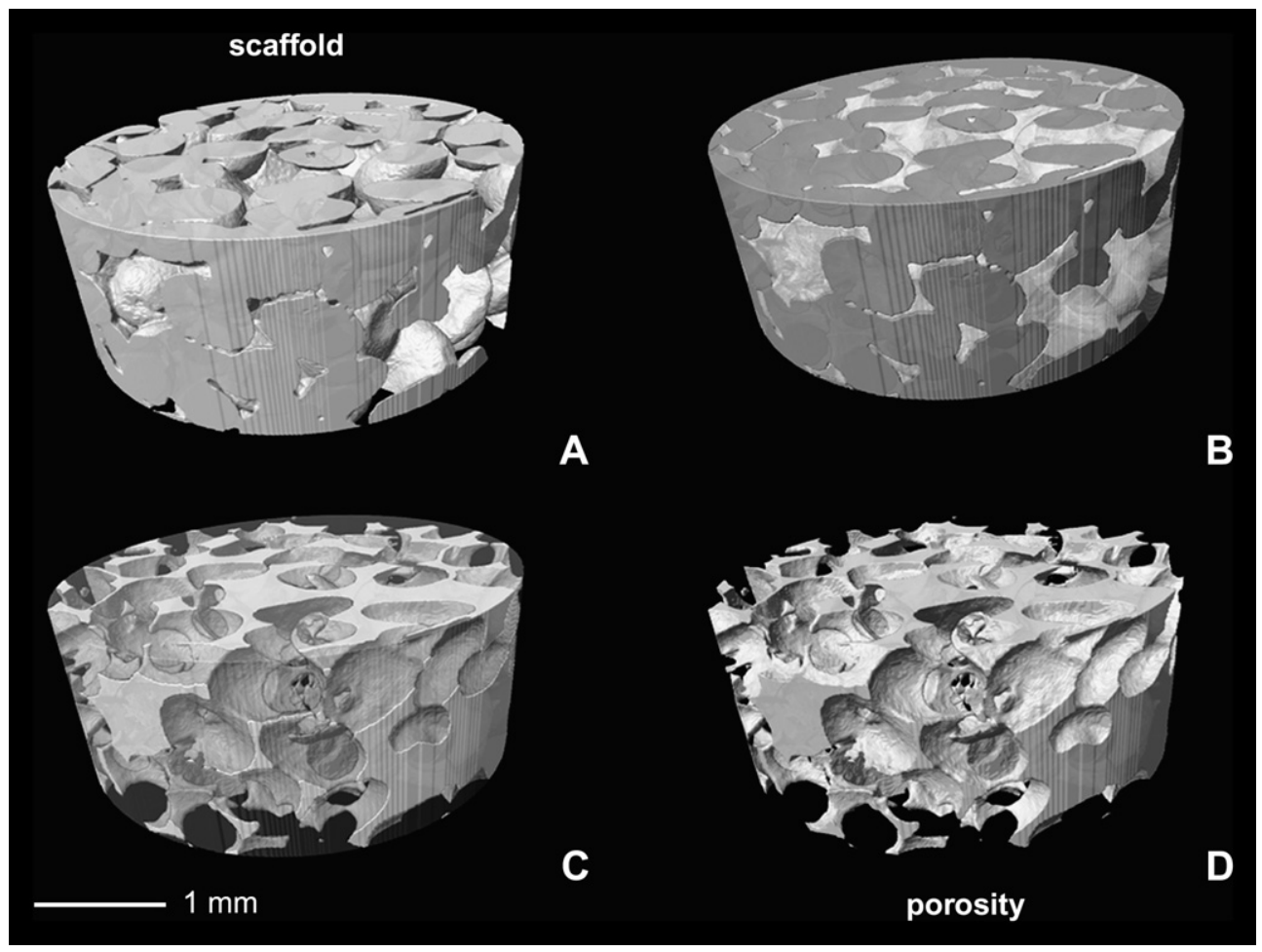

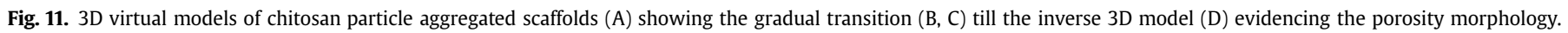

particle aggregation method process. This was achieved due to the bioadhesive character of the chitosan polymer that resulted in the merging of adjacent particles at their contact points to form the chitosan porous 3D matrices. The cross-sections from the scaffolds' bulk stained with eosin (Fig. 13) showed clearly the bonding areas on the particle surfaces. This chitosan particles bonding leads to a very stable interface between the particles which assures the mechanical integrity and stability of the developed scaffolds.

\subsection{In vivo biocompatibility}

The non-cytotoxic behaviour of the chitosan particle aggregated scaffolds was evaluated in previous studies [21]. In vitro tests showed that the chitosan particle aggregated scaffolds were not cytotoxic to L929 fibroblast cell line and to human adipose stem cells [21]. However, it is critical to evaluate the scaffolds' performance in an in vivo environment when proposing scaffolds to be used in biomedical applications, namely tissue engineering. In vivo parameters such as inflammatory response, tissue ingrowth, vascularization potential and overall host response gives the correct evidence to consider the biofunctionality of scaffolds. This is one of the obvious key requirements for tissue engineering applications.

After intramuscular implantation of the developed scaffolds, no systemic or regional surgical complications were seen in any of the rats. Under optical microscopy it is clear that the chitosan scaffolds architecture allowed for tissue ingrowth, being the feature more evident for longer periods of implantation (Figs. 4 and 5). Micro-CT also allowed assessing the increase of spatial and temporal tissue

Table 2

Interconnectivity calculated with different voxels size

\begin{tabular}{lll}
\hline Limit voxels & $\begin{array}{l}\text { Limit voxel } \\
\text { size }(\mu \mathrm{m})=\text { pore size }\end{array}$ & $\begin{array}{l}\text { Interconnectivity (\%) } \\
(\text { mean } \pm \text { SD) }\end{array}$ \\
\hline 2 & 17.58 & $99.27 \pm 0.89$ \\
4 & 35.16 & $96.52 \pm 1.55$ \\
6 & 52.74 & $94.99 \pm 1.41$ \\
\hline
\end{tabular}

ingrowth into the scaffolds, as shown in the 2D X-ray cross-sections from the bulk of the implants (Fig. 5). The increase in the connective tissue ingrowth provides a clear evidence that the interconnectivity of the developed scaffolds plays a key role in this process. The influence of interconnectivity on the tissue ingrowth was already demonstrated by Otsuki et al. [10] by studying the effect of narrow pore throats on bone and tissue differentiation using bioactive porous titanium implants and micro-CT. The study revealed that narrow pore throats (e.g., low interconnectivity with low pore size limitation for its calculation) were inhibiting tissue differentiation in pores [10].

There is also a clear macroscopic indication of the presence of a vascularized tissue after 12 weeks of implantation that was further confirmed by the immunohistochemistry analyses. Again, scaffolds interconnectivity seems to be favourable for a good integration of the scaffolds in the host tissue, promoting the vascularization of the scaffolds which is critical in tissue engineering applications for cell growth, tissue ingrowth and nutrients flow. For tissue differentiation, vascularization is an important factor, and narrow pore interconnectivity may inhibit adequate vascularization [10].

After implantation of a medical device such as a biomaterial, the host tissue will inevitably be traumatized by the implantation procedure $[29,30]$ triggering an inflammatory response. An inflammatory response triggered by chitosan particle aggregated scaffolds was observed after 1, 2 and 12 weeks of intramuscular implantation in rats. One week after the implantation of the chitosan particle aggregated scaffolds, PMNs were the predominant inflammatory cell type (Figs. 6A and 7B). These cells are normally recruited from blood circulation whenever there is tissue damage and the initiation of the process of wound healing. In less extent, lymphocytes (Fig. 8B) and macrophages (Fig. 6A) were observed, following again the typical features of the implantation of foreign bodies $[29,30]$. The type of inflammatory infiltrate found in the surrounding tissue of the implanted chitosan particle aggregated scaffolds is the type of infiltrate expected when a foreign body is 


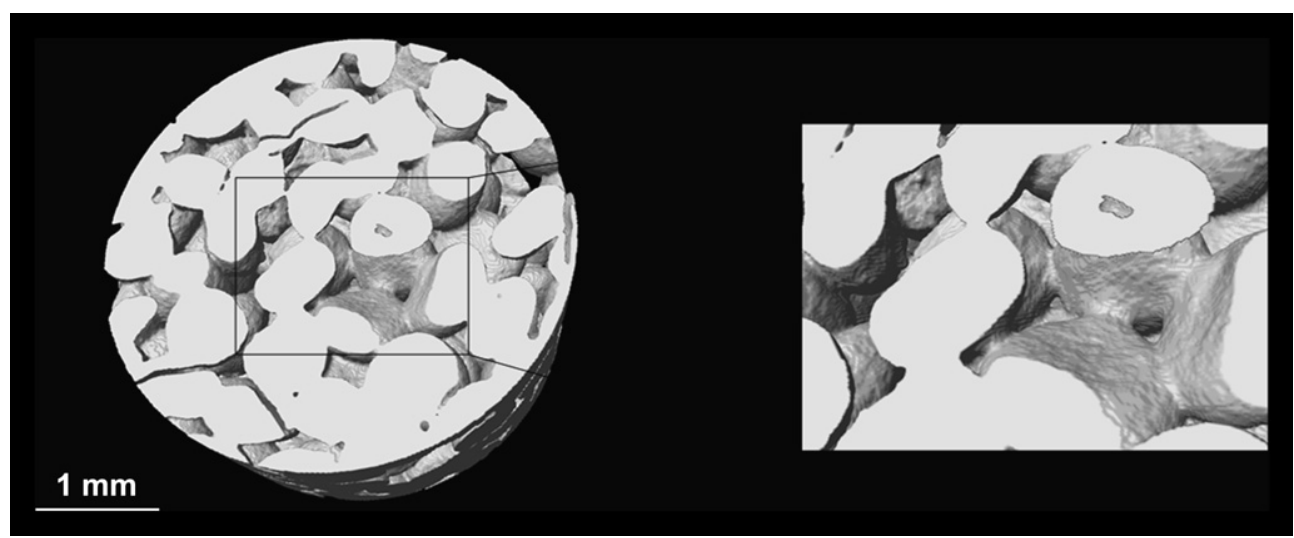

Fig. 12. 3D virtual models of chitosan particle aggregated scaffolds evidencing the interface between the particles.

implanted in a host [29,30], showing a normal and mild inflammatory reaction towards the implants. Few new blood vessels can be found at this stage (Figs. 9B and 10B). The neo-vascularization observed at this stage identified by the $\mathrm{vWF}$ expression (Fig. 9B) is due to the initially formed clot in part to the inflammatory process that brings the leukocytes in circulation to the site of implantation after the surgical procedure. Even though, it is considered very important for the nutrition of the new tissue that is expected to grow in the spaces between the particles forming the scaffold.

In contrast with the 1 week of implantation, after 2 weeks, the principal cell type in the scaffold and surrounding tissue were without any doubt lymphocytes. These cells start to appear when the acute inflammation progresses to chronic inflammation, around the 2nd week after the tissue injury and if the damaging agent persists in the site [31]. When the implant has dimensions which the phagocytic cells are not able to phagocytose and the macrophages may fuse, forming foreign body giant cells [32]. Therefore, the observed reaction coincides with the patterns of a mild foreign body reaction [32]. The initially formed clot (resulting from the implantation procedure and consequent cascades of events for wound healing) is now less evident (Fig. 9C).

After 12 weeks in the intramuscular environment, some foreign body giant cells were observed near the implanted particle aggregated chitosan scaffolds. Although some PMNs and lymphocytes were observed, the type of reaction showed to be non-granulomatous [33]. This type of inflammatory infiltrate, where mononuclear cells clearly predominate, is quite typical for foreign body reactions [32] and shows a normal response from the host to the chitosan particle aggregated scaffolds [32].

The neo-vascularization of the spaces between the particle forming the chitosan-based scaffolds was also achieved (Figs. 9 and 10). One week after implantation, few new blood vessels were observed, responsible for the recruitment of leukocytes to the site of implantation. In early stages of inflammation and wound healing process, neo-vascularization is an important feature, since it allows the adequate nutrition of the new connective tissue that will grow around and between the scaffold structure. With the increasing need of nutrition, justified by the increase of extracellular matrix formation, the density of vascularization increased as well after 2 and 12 weeks of implantation. After these periods of implantation, it was possible to observe that the scaffolds induced a marked angiogenic response promoted also by the high scaffolds interconnectivity (Figs. 9C, D, and 10C, D).

The neo-vascularization of the implants created by new blood vessels formation was clear even after only 2 weeks of implantation, becoming more significant as the implant remained for a longer period of time, showing that the scaffolds characteristics were favourable to the integration in the host tissue. These blood vessels established and developed as seen for 12 weeks after scaffolds implantation. In fact, the vascularization is another critical factor for a successful approach in tissue engineering. The chitosan scaffold architecture has shown its ability to allow for cells ingrowth, and subsequent migration into and through the matrix. This was accomplished given that the 3D materials enabled the mass transfer of nutrients and metabolites, and provided sufficient space for development and later remodelling of the organized tissue.

The formation of blood vessels developing inside the scaffold was a remarkable result since no angiogenic growth factor or previously seeded angiogenic cells were used in this study. This kind of evidence leads one to speculate that the good in vivo performance of the chitosan-based scaffolds produced by particle aggregation can be still further improved by using any angiogenic growth factor or cells important in vasculature. These results were also observed by Ehrenfreund-Kleinman et al. [34] for chitosan/arabinogalactanbased sponges where the vascularization became clearer for 11
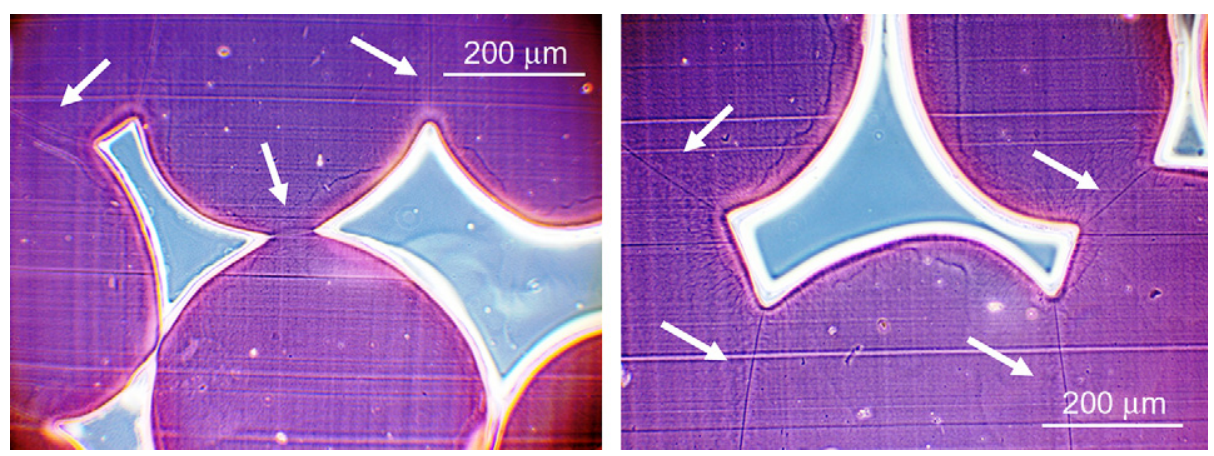

Fig. 13. Cross-sections of chitosan particle aggregated scaffolds stained with eosin showing the particles' interface indicated by the arrows. 
weeks of implantation period as detected by light microscopy and magnetic resonance imaging. The generation of extracellular matrix by the connective tissue cells between the spaces of the particle aggregated chitosan scaffolds was also identified (Fig. 6C). Furthermore, the immunolabelling of smooth muscle actin (SMA) allowed, at the same time, for the observation of vessels' ingrowth, since it is one of the constituents of the vessel's walls. At 1 week of implantation, it was possible to observe some extracellular matrix form a still disorganized network between the particles of the scaffold (Fig. 6A). This network increased the amount of extracellular matrix and organization along the time of implantation. These overall features show that the implanted particle aggregated chitosan scaffolds are well tolerated by the host. Furthermore, it was shown that the scaffolds architecture, namely pore interconnectivity, is favourable for cell ingrowth and subsequent production of extracellular matrix by the connective tissue cells.

The overall host response elicited by the intramuscular implantation of chitosan particle aggregated scaffolds was a mild and expected inflammatory response. It started with the normal acute inflammation initiated by the surgical procedure of implantation, noticed at the first week progressing to the normal chronic inflammation usually observed in the implantation of foreign bodies $[31,32]$. The increasing of neo-vascularization, as well as the presence of smooth muscle actin and the enhanced organization of the extracellular matrix with the time of implantation, demonstrates the good integration and scaffolds' biofunctionality. The high interconnectivity of the developed chitosan particle aggregated scaffolds might also play a key role in the scaffold integration with vascularization from the host tissue.

\section{Conclusions}

In this study, we manage to fabricate chitosan scaffolds based on a particle aggregation technique. These scaffolds have shown to fulfill three main key requirements for tissue engineering scaffolding: morphological adequacy, mechanical stability and in vivo functional biocompatibility. Micro-CT allowed for an accurate morphometric characterization showing that the developed scaffolds had an adequate pore size range and an interconnected porous structure assuming a relevant interconnection limit. Furthermore, porosity morphology will allow for tissue ingrowth resembling those of trabecular bone, if this application is considered. In addition to all this, the mechanical properties demonstrated the scaffolds stability and suitability for load-bearing bone tissue engineering applications. From a biological point of view, it is possible to conclude that the chitosan particle aggregated scaffolds are suitable structures to allow tissue ingrowth with an induced minimal inflammatory response by the host in an intramuscular site. Furthermore, neo-vascularization, as well as the enhanced organization of the extracellular matrix, with the implantation time confirms the scaffolds' biofunctionality. It is concluded that chitosan scaffolds produced by a particle aggregation methodology are morphological and mechanically adequate for their aimed applications, with demonstrated in vivo functionality. Consequently, they are without doubts potential candidates for being used in load-bearing tissue engineering applications.

\section{Acknowledgments}

The authors would like to acknowledge the Portuguese Foundation for Science and Technology for the PhD Grant to Patrícia B. Malafaya (SFRH/BD/11155/2002). This work was partially supported and carried out under the scope of the European STREP Project HIPPOCRATES (NMP3-CT-2003-505758) and European NoE EXPERTISSUES (NMP3-CT-2004-500283). The authors also thank Prof. Heinz Redl for the collaboration in the in vivo studies, as well as Bernhard Höring for the surgical procedures both from LBI, Austria and João Oliveira from 3B's Research Group, Portugal for the initial assistance with the DMA equipment.

\section{References}

[1] Hollister SJ. Porous scaffold design for tissue engineering. Nat Mat 2005;4(7): 518-24.

[2] Hutmacher DW, Schantz JT, Fu CX, Cheng LK, Lim TTC. State of the art and future directions of scaffold-based bone engineering from a biomaterials perspective. J Tissue Eng Regen Med 2007;1(4):245-60.

[3] Mano JF, Silva GA, Azevedo HS, Malafaya PB, Sousa RA, Silva SS, et al. Natural origin biodegradable systems in tissue engineering and regenerative medicine: present status and some moving trends. J R Soc Interface 2007;4(17): 999-1030.

[4] Noth U, Rackwitz L, Heymer A, Weber M, Baumann B, Steinert A, et al. Chondrogenic differentiation of human mesenchymal stem cells in collagen type I hydrogels. J Biomed Mater Res A 2007;83(3):626-35.

[5] Park H, Temenoff JS, Tabata Y, Caplan AI, Mikos AG. Injectable biodegradable hydrogel composites for rabbit marrow mesenchymal stem cell and growth factor delivery for cartilage tissue engineering. Biomaterials 2007;28(21): 3217-27.

[6] Mastrogiacomo M, Scaglione S, Martinetti R, Dolcini L, Beltrame F, Cancedda R, et al. Role of scaffold internal structure on in vivo bone formation in macroporous calcium phosphate bioceramics. Biomaterials 2006;27(17):3230-7.

[7] Zhang Y, Ni M, Zhang M, Ratner B. Calcium phosphate-chitosan composite scaffolds for bone tissue engineering. Tissue Eng 2003;9(2):337-45.

[8] Bobyn JD, Pilliar RM, Cameron HU, Weatherly GC. The optimum pore size for the fixation of porous surfaced metal implants by the ingrowth of bone. Clin Orthop Relat Res 1980;150:263-70.

[9] Lu JX, Flautre B, Anselme K, Hardouin P, Gallur A, Descamps M, et al. Role of interconnections in porous bioceramics on bone recolonization in vitro and in vivo. J Mater Sci Mater Med 1999;10(2):111-20.

[10] Otsuki B, Takemoto M, Fujibayashi S, Neo M, Kokubo T, Nakamura T. Pore throat size and connectivity determine bone and tissue ingrowth into porous implants: three-dimensional micro-CT based structural analyses of porous bioactive titanium implants. Biomaterials 2006;27(35):5892-900.

[11] van Lenthe GH, Hagenmuller $\mathrm{H}$, Bohner M, Hollister SJ, Meinel L, Muller R. Nondestructive micro-computed tomography for biological imaging and quantification of scaffold-bone interaction in vivo. Biomaterials 2007;28(15): 2479-90.

[12] Ho ST, Hutmacher DW. A comparison of micro-CT with other techniques used in the characterization of scaffolds. Biomaterials 2006;27(8):1362-76.

[13] Feldkamp LA, Goldstein SA, Parfitt AM, Jesion G, Kleerekoper M. The direct examination of three-dimensional bone architecture in vitro by computed tomography. J Bone Miner Res 1989;4(1):3-11.

[14] Hildebrand T, Laib A, Muller R, Dequeker J, Ruegsegger P. Direct three-dimensional morphometric analysis of human cancellous bone: microstructural data from spine, femur, iliac crest, and calcaneus. J Bone Miner Res 1999;14(7): 1167-74.

[15] Mastrogiacomo M, Komlev VS, Hausard M, Peyrin F, Turquier F, Casari S, et al. Synchrotron radiation microtomography of bone engineered from bone marrow stromal cells. Tissue Eng 2004;10(11-12):1767-74.

[16] Mathieu LM, Mueller TL, Bourban P-E, Pioletti DP, Muller R, Manson JAE. Architecture and properties of anisotropic polymer composite scaffolds for bone tissue engineering. Biomaterials 2006;27(6):905-16.

[17] Ma PX. Biomimetic materials for tissue engineering. Adv Drug Deliv Rev 2008; 60(2):184-98.

[18] Malafaya PB, Silva GA, Reis RL. Natural-origin polymers as carriers and scaffolds for biomolecules and cell delivery in tissue engineering applications. Adv Drug Deliv Rev 2007;59(4-5):207-33.

[19] Kim IY, Seo SJ, Moon HS, Yoo MK, Park IY, Kim BC, et al. Chitosan and its derivatives for tissue engineering applications. Biotechnol Adv 2008;26(1): $1-21$.

[20] Nettles DL, Elder SH, Gilbert JA. Potential use of chitosan as a cell scaffold material for cartilage tissue engineering. Tissue Eng 2002;8(6):1009-16.

[21] Malafaya PB, Pedro A, Peterbauer A, Gabriel C, Redl H, Reis RL. Chitosan particles agglomerated scaffolds for cartilage and osteochondral tissue engineering approaches with adipose tissue derived stem cells. J Mater Sci Mater Med 2005;16(12):1077.

[22] Garner E, Lakes R, Lee T, Swan C, Brand R. Viscoelastic dissipation in compact bone: implications for stress-induced fluid flow in bone. J Biomech Eng 2000; 122(2):166-72.

[23] Barker MK, Seedhom BB. Articular cartilage deformation under physiological cyclic loading - apparatus and measurement technique. J Biomech 1997; 30(4):377-81.

[24] Hristov M, Weber C. Endothelial progenitor cells: characterization, pathophysiology, and possible clinical relevance. J Cell Mol Med 2004;8(4):498-508.

[25] Huang Y, Onyeri S, Siewe M, Moshfeghian A, Madihally SV. In vitro characterization of chitosan-gelatin scaffolds for tissue engineering. Biomaterials 2005;26(36):7616-27.

[26] Yin Y, Ye F, Cui J, Zhang F, Li X, Yao K. Preparation and characterization of macroporous chitosan-gelatin/beta-tricalcium phosphate composite scaffolds for bone tissue engineering. J Biomed Mater Res A 2003;67A(3):844-55. 
[27] Jiang T, Abdel-Fattah WI, Laurencin CT. In vitro evaluation of chitosan/poly(lactic acid-glycolic acid) sintered microsphere scaffolds for bone tissue engineering. Biomaterials 2006;27(28):4894-903.

[28] Tuzlakoglu K, Alves CM, Mano JF, Reis RL. Production and characterization of chitosan fibers and 3D fiber mesh scaffolds for tissue engineering applications. Macromol Biosci 2004;4(8):811-9.

[29] Babensee JE, Anderson JM, McIntire LV, Mikos AG. Host response to tissue engineered devices. Adv Drug Deliv Rev 1998;33(1-2):111-39.

[30] Williams DF. On the mechanisms of biocompatibility. Biomaterials 2008; 29(20):2941-53.
[31] Lickorish D, Chan J, Song J, Davies JE, Kirkpatrick J, Bongrand P. An in-vivo model to interrogate the transition from acute to chronic inflammation. Eur Cell Mater 2004;8:12-20.

[32] Luttikhuizen DT, Harmsen MC, Van Luyn MJA. Cellular and molecular dynamics in the foreign body reaction. Tissue Eng 2006;12(7):1955-70.

[33] Stevens A, Lowe J, Young B. Wheater's basic histopathology: a colour atlas and text. Edinburgh: Churchill Livingstone; 2002.

[34] Ehrenfreund-Kleinman T, Gazit Z, Gazit D, Azzam T, Golenser J, Domb AJ. Synthesis and biodegradation of arabinogalactan sponges prepared by reductive amination. Biomaterials 2002;23(23):4621-31. 